\title{
Discovery by Serendipity: a new context for an old riddle
}

\author{
Pio García \\ Universidad Nacional de Córdoba
}

\begin{abstract}
In the last years there has been a great improvement in the development of computational methods for combinatorial chemistry applied to drug discovery. This approach to drug discovery is sometimes called a "rational way" to manage a well known phenomenon in chemistry: serendipity discoveries. Traditionally, serendipity discoveries are understood as accidental findings made when the discoverer is in quest for something else. This 'traditional' pattern of serendipity appears to be a good characterization of discoveries where "luck" plays a key role. In this sense, some initial failures in combinatorial chemistry are frequently attributed to a naï appropriation of a "serendipity model" for discovery (a "serendipity mistake"). In this paper we try to evaluate this statement by criticizing its foundations. It will be suggested that the notion of serendipity has different aspects and that the criticism to the first attempts could be understood as a "serendipity mistake". We will suggest that "serendipity" strategies, a kind of blind search, can be seen sometimes as a "genuine part" of scientific practice. A discussion will ensue about how this characterization can give us a better understanding of some aspects of serendipity discoveries.
\end{abstract}

\section{Introduction}

Since the 1980s a new approach called "combinatorial chemistry" has been used to synthesize and screen new compounds. This approach was seen as a promising tool for developing new compounds and also for discovering new drugs. Actually, "combinatorial chemistry" is a general term used to refer to different techniques and procedures that employ a kind of "brute force" strategy for generating or screening chemical compounds.

Considering the big impact that this approach had in the 1990s, it is now common to read critical reviews that adopt a historical perspective about faults and flaws in the first combinatorial chemistry approaches. By criticizing these first attempts some researchers intend to correct the supposed previous 'mistakes'; the majority of which seem to include the use of a "serendipitous approach".

1 There are some criticisms related to the whole combinatorial approach and not only to the first approach, which is not considered here. For example, Horrobin [7] has suggested that the "artificial", or in vitro approach, of combinatorial chemistry did not take into account the complexity of in vivo situation. In combinatorial chemistry the proteins are studied as 
Apparently, those reviews implied that serendipity somehow represents the irrational part of the discovery process. This rough idea is not foreign in philosophy either. Serendipity discoveries are most of the time part of historical accounts rather than of philosophical reconstructions.

However, in this paper we will argue that serendipity is more than just the irrational part of certain scientific discoveries. It cannot be denied that there are "accidental" aspects in serendipity that may sometimes be crucial, however those are not the aspects that combinatorial chemists consider as "serendipity mistakes".

The word serendipity - including in the expression "serendipity mistake" - is actually used by the scientists themselves to refer to "the first approach" in combinatorial chemistry. The expression "serendipity mistake" was coined by those scientists as a way to describe the principal error committed by the first researchers in combinatorial chemistry.

In the first section of this paper we will analyze the notion of serendipity through schematic historical cases to then identify what is meant to be the "irrational" side of serendipity by the researchers of combinatorial chemistry. In the second section, we will present some evidence regarding the beginnings of combinatorial chemistry and the reasons that led to the partial characterization of its methodological activity in terms of "serendipity". Finally, we will describe how some aspects of serendipity are used as a strategy in scientific practices.

\section{What is serendipity?}

Although science is essentially considered a rational enterprise, there are some historical accounts of scientific discoveries where the crucial role seems to be played by "chance" or by accidental circumstances. Those "happy findings" are generally called discoveries by 'Serendipity'.

There are several reports of serendipity: some from a historical point of view [20], others from a philosophical point of view [12], and yet others from a combination of perspectives [19]. But if we try to obtain a characterization of scientific practices, it is good to start by seeing some examples.

Stories about discoveries by serendipity have been frequently reported in chemistry and biochemistry. A commonly cited example is from the work of Pasteur on chirality. In 1848 at the beginning of his career, Pasteur tried to solve a problem that Eilhard Mitscherlich had noticed: a salt of tartaric acid formed in a wine cask was optically active (dextrorotatory), whereas a salt of racemic acid found in another stage of the fermentation process and with the same composition was optically inactive [6]. Pasteur examined under a microscope a sample of racemic acid salt and he saw two kinds of crystals with similar shapes but nonsuperimposable. He separated the crystals on the left and right with tweezers. The solution of each kind of crystal rotated the light in opposite directions, dextrorotatory and levorotatory.

This very schematic account is a description of normal and regular research. But Pasteur himself later recognizes the serendipitous character of this discovery because

crystals or in an aqueous solution and not in a lipid membrane (in vivo condition), so some hits could be found through combinatorial chemistry that would not be effective in vivo. 
of several circumstances that made it possible. The sodium ammonium salt of racemic acid is almost the only salt from this acid with crystals that can be manipulated in a mechanical way [20]. It is also pointed out that this substance is crystallized only at a temperature below $26^{\circ}$ Celsius, and the night before Pasteur's observation he had left the racemic acid sample in an open window. In this case, serendipity is understood mainly through the accidental circumstances that surround it. Anecdotal accounts of serendipitous discoveries emphasize this idea. From this perspective, serendipity is just another name for 'casual findings' and could only be explained by historical or sociological accounts.

However, there is another aspect to be considered beyond accidental circumstances in serendipitous discoveries. Pasteur noticed something that chemists before him had overlooked. Both aspects, accidental circumstances and sagacity, can be summarized by Pasteur's famous dictum: "in the field of observation, chance favors only a prepared mind". In this tension between "chance" and a "prepared mind", the latter is considered the rational side of a serendipitous discovery.

The discovery of new drugs is a good source of numerous examples of "a prepared mind". Possibly the best known example of a serendipitous drug discovery is penicillin. The discovery was made by Sir Alexander Fleming when he realized that a contaminated sample in a Petri dish could be something other than a mistake. He was in the middle of research on influenza when he saw a culture of Staphylococcus bacteria accidentally contaminated by a mold. Instead of throwing the sample away, he did some research. When Roberts [20] retells this story he points out that Fleming's previous experience with lysozyme could have been important in his decision to conduct research on that dish ${ }^{2}$. The moral of this story seems to be the importance of "skillful perception". It is clear that Fleming's discovery was surrounded by "happy" accidents, but it is also true that this achievement was possible because of a well "prepared mind".

The last two characterizations, on the other hand, do not exhaust serendipity's meaning. The case of salicylic acid that was first synthesized for infections without success and then modified and used for pain and fevers is sometimes cited; the Minoxidil case is also reported, which was mainly used for high blood pressure, when its uses as an aid for hair growth were accidentally discovered. In those situations, serendipity is regarded as an unnoticed application of a previously known drug. A very similar pattern is found with the discovery of urea synthesis. In 1828 Friedrich Wöhler tried to prepare pure ammonium cyanate from potassium cyanate and ammonium sulfate, but what he obtained was white crystals that were actually urea. This is how Wöhler synthesized an organic compound, urea, in his laboratory for the first time. He was looking for one thing and obtained something different. In all of those cases stressing the "prepared mind", serendipity is seen as a kind of search, which is not only another meaning of serendipity, but the original meaning of it.

Serendipity discoveries are traditionally understood as accidental findings made when the discoverer is investigating something else. From this source, serendipity discoveries are usually described as looking for one thing and finding another. This can be called the 'traditional' pattern of serendipity discovery and it seems to be the

2 But in Fleming's own words these previous experiences can be seen as a reason for discarding those samples. 
"original" meaning. As is widely known, the word "Serendipity" came from Horace Walpole's letter written on January 28, 1754. In this letter, Walpole cited an old "silly fairy tale" about the three princes of Serendip that made discoveries through "accidents and the sagacity of things which they were not in quest of" [19]. Walpole called these kinds of discoveries "instances of accidental sagacity" highlighting the accidental finding: "no discovery of a thing you are looking for comes under this description" (p.2).

From these examples we can see that serendipity is not referring to a simple phenomenon but to different aspects of various discoveries beyond the accidental circumstances. There are at least four 'aspects' of serendipity: (a) accidental circumstances, (b) a kind of search (c) "prepared mind" or "sagacity" and (d) finding a thing that is not being sought out. As we have seen, aspect (d) is the original one, however, serendipity seems to be defined in reference to historical cases - in an indexical way - and therefore the aspects (a), (b) and (c) may be considered as "genuine" parts of that notion. But evidently, these first three aspects should be seen as complementary. None of them individually can typify a discovery by serendipity, as is the case for aspect (d). In this way, the (a), (b) and (c) aspects can only refer to serendipity when they are combined each other. Characterizing the notion of serendipity in terms of "aspects" allows us to differentiate the various discovery cases. As is shown by the examples, what differentiates one serendipity case from another, on occasion, is the presence or absence of one aspect, and in another the relevance of the aspects involved. But in any form it seems clear that the notion of serendipity should not be circumscript in the sense of (d). This is a truly important aspect of the notion of serendipity - and in the genetic sense the "original one". But rejecting the others serendipity's aspects seem to lead to the loss of descriptive capacity of historical discoveries. This is probably a consequence of the fact that notions of serendipity have been constructed by pointing out the paradigmatic cases.

Of the aspects which characterize the notion of serendipity, (b) a kind of search, (c) prepared mind and (d) traditional perspective seem especially relevant to address the problems we are dealing with in combinatorial chemistry. With the aim of achieving a vision that is adequate for the understanding of the discovery, it could be useful to look at these aspects in terms of problem solving. The differences between the aspects of serendipity can be articulated by widely used problem solving metaphors. A problem solving activity can be represented by a "satisfaction" metaphor and in other cases by a "search" metaphor. In the first metaphor, problem solving activity is seen as a process of narrowing down an original set by means of the satisfaction of defined constrains. With the second metaphor, problem solving activity is understood as a movement in a problem space looking for a solution state. The same problem solving task can be described by both metaphors although they do not stress the same features of problem solving activity.

We suggest that the "prepared mind" approach endorses a "satisfaction" metaphor and the "kind of search" or the "traditional perspective", stressing the task of finding something by accident, can be understood as endorsing a "search" metaphor.

From the previous discussion it is not directly obvious which aspect of serendipity is referred to when researchers say that the "rational turn" of combinatorial chemistry can be understood as a 'departure' from serendipity. 


\section{Combinatorial chemistry and serendipity: the first approach}

Combinatorial methods in chemistry are usually related to techniques and devices for synthesizing large amounts of compounds and for screening the generated materials. The first methodological approach is called combinatorial synthesis and the second is called high-throughput screening. More specifically, according to Valerie Gillet, combinatorial synthesis "refers to the synthesis of large numbers of compounds in parallel where product molecules are formed as combinations of available reagents or buildings blocks" [17] (p. 617); high-throughput screening "is an automated process whereby a large number of compounds $\left(10^{4}-10^{5}\right)$ are rapidly screened for biological activity" [17] (p. 617).

Combinatorial chemistry techniques are at times used for developing new materials or for discovering new properties of already known materials, such as polymers [20], but they are principally used for drug discovery and development. Merrifield's work on solid-phase synthesis done at the beginning of the 1960's is commonly referred to as one of the origins of combinatorial synthesis [18]. Today, there is also a solutionphase approach to peptide synthesis which has other virtues and other problems [3]. Even so, Merrifield's work remains important since it represents the first steps taken towards the automation of a whole synthetic process. Automation, along with other conditions like miniaturization, is part of what we call the "first approach" in combinatorial synthesis ${ }^{3}$. With this technique, Merrifield tried to overcome the "practical limits" of time and yield in the synthesis of polypeptides by proposing a method of "greatest simplicity and efficiency" [18] (p. 178).

This approach in combinatorial synthesis, more than just a new methodology, represents an automation of traditional methodologies with the help of new techniques. To reinforce this point, we can quote researchers in this field that described the differences between a classical approach and a combinatorial approach by the kind of process involved when a chemist synthesizes a compound. In the first case - classical approach- the chemists usually use a serial process and in the second case the chemists use an automatic and parallel process [3].

The "first approach" depended mainly on techniques and devices that allow for a larger and faster output of useful compounds, leaving the question open regarding search restrictions. Therefore, the "first approach" used an unrestricted or a kind of very powerful blind search. This process is blind in the sense that it can be considered a "generate-and-test-all" process and thus is sometimes compared to a serendipitous means to obtain new compounds or drugs.

It is quite common for scientists to describe the methodology used in the "first approach" in terms of "serendipity". Combinatorial chemistry and HTS would thus be "the purely serendipity-based 'brute-force' methods" (4). It seems normal, therefore, that those very scientists involved in developing these methods describe the "first approach" in terms of serendipity.

Apparently, the intuition behind "first approach" is that serendipity, understood in this way, can be considered as the engine in the drug discovery process. As a consequence, the opinion at that time was that "simply increasing throughput would be sufficient to improve chances of finding novel bioactive compounds" [17] (p. 617).

3 The title of this first article is: "Automated Synthesis of Peptides: solid-phase peptide synthesis, a simple and rapid synthetic method, has now been automated" [18]. 
What's more, the unrestricted or blind search aspect from the "first approach" is considered as an inappropriate methodology for what some have denominated "the rational turn" in combinatorial chemistry. And, sometimes, this assessment against the "first approach" is presented in terms of "serendipity". In sum, the "first approach" was inadequate because it assumed a type of methodology related to discoveries by "serendipity".

Thus, in Huser's High-throughput screening in drug discovery [10], for example, a strategy designated as "systematic serendipity" is presented to methodically discover -- no longer by mere trial and error --the "potential side attributes of small molecule HTS "hits" and advanced compounds" [10] p.318). Kubinyi has also said that "whereas the very first syntheses of huge libraries of peptides, peptide derivatives, peptoids, and other peptidomimetics more or less followed Swift's principle of a random combination of building blocks, the design of focused libraries is now in the foreground" [13].

The question then arises of whether or not the use of the word serendipity is adequate to characterize the "first approach" and, in a negative sense, the subsequent methodological changes. One could make the case that this use of the word serendipity was merely metaphorical — or even rhetorical — and consequently does not say much regarding these methodological changes.

\section{The "rational turn" in combinatorial chemistry}

The negative relationship between economic investment and the "first approach" results was not expected. From this practical failure some chemists derived a more general criticism of combinatorial chemistry: "It soon became apparent that a "makeand-test-all' approach was neither practical nor possible" [17] (p. 617). The "impossibility" included in the "first approach" is seen here as a simple corollary of their combinatorial nature. There are very simple examples that are used to show the combinatorial problem. Even with a simple library with a diamine three-component, there are $10^{12}$ potential products using commercially available reagents.

Profuse literature exists about changes in combinatorial chemistry from the 1990s perspective, which is usually summarized as a more "rational" comprehension of the combinatorial chemistry task; we call this change the "rational turn" in combinatorial chemistry. This "rational turn" can be understood by surveying how an intuitive and broad notion of "design" is used in combinatorial chemistry. By implying an intentional and a planned aspect, this design notion has an obvious rational meaning. One of the most cited examples of the design notion used by the "rational turn" is structure-based drug design [21], in which combinatorial methods for modifying and improving drug leads are used: "structure-based drug design is supported by computer programs for the automated superposition (alignment) of molecules, for flexible docking of ligands and for de novo design of ligands that fit a binding site in shape and complementarity of their physicochemical properties" [13] (p. 9)

Another kind of "design" included in this new combinatorial chemistry is library design, both physical and virtual. Considering the large possible chemistry space, it is 
common to make a selection of compounds for screening. Among the methods used for this task a kind of "diversity" selection is very common [13] (p 87).

Finally, another sign of the "rational" turn is the in silico approach to combinatorial chemistry. Using computers in combinatorial chemistry further simplified the intended automation associated with earlier combinatorial synthesis and also made explicit some knowledge about compound representation and their relevant properties. With computational strategies, a previous selection is made of the compounds that are going to be synthesized. The selection depends on the objectives of the experiment. In the case of drug discovery, taking into account properties like absorption, metabolism, excretion or a version of Lipinski's rule of five, are usually considered a good starting point. In more general terms, virtual screening, or in silico screening, "refers to the use of computational techniques to select compounds, either from existing libraries... or from virtual libraries that represent the compounds that could potentially be made via combinatorial synthesis" [17] (p. 618).

The "rational" approach to combinatorial chemistry as represented by different techniques, assumed a perspective that moves away from an unrestricted search, while the "first approach" in combinatorial chemistry identifies serendipity with an unrestricted or blind search. The negative assessment of the "first approach" implied by the "rational turn" suggests that the aspect of serendipity considered here is neither related to circumstances nor the "prepared mind", but to a particular kind of search. As a consequence, if we relate the metaphors that we proposed earlier - satisfaction and search - to Pasteur's dictum, the results seem to be that the satisfaction metaphor is on the side of rationality and the search metaphor represents the irrational part of serendipity.

This leads to two questions: the first is related to whether or not the notion of "serendipity mistake", as articulated by those who criticize "the first approach" in combinatorial chemistry, is used in a metaphorical sense or it could be used as an accurate application of serendipity's concept. The second question is whether the particular kind of search that seems to characterize "the first approach" is always an irrational strategy.

Related to the first question: if, as we have seen, one of the aspects through which the notion of serendipity can be seen is a blind search - in conjunction with other aspects - then the attribution of the term "serendipity" by those who criticize the first intents in combinatorial chemistry seems to be a proper use of serendipity's concept. It is probable that this use is motivated by the number of the cases of new drug discoveries where "random" aspects are involved; nonetheless, if our analysis of the notion of serendipity is correct, then a kind of "blind search" has to be considered an actual aspect of serendipity. On the other hand, the question regarding the evaluation of the "first approach's" blind search requires a separate analysis.

\section{The "serendipity mistake" reconsidered: blind search}

The rational turn's negative evaluation of the "first approach" seems motivated in principle by practical questions: the economic investment made in this research program did not produce the expected results. Furthermore, the emergence of new 
techniques for drug discovery that adopted a design approach used combinatorial chemistry procedures, however within very different constrains. But can we generalize these considerations?

It is assumed here that any kind of blind search is always irrational and that science is actually a rational enterprise. But the previous assumption about blind searches can be deflated by taking into account a creative context and considering scientific practices.

First, it can be argued, following Donald Campbell [5], that a kind of blind search is a good strategy when we are dealing with creativity. There is a classical discussion in problem solving about how to obtain genuine novelties as a solution to a problem if the problem's definition involves the description of an already known objective -the classical Meno's Paradox. An "unguided" or blind search is the answer to this paradox that some philosophers proposed because the solution to a problem is not necessary anticipated and, at least in principle, the field of drug discovery belongs within the context of creativity. Second, it seems that in scientific practices sometimes a kind of blind search can be a genuine strategy. A "surprising" finding in science is usually described as serendipity because of the "blindness" assumed in the discoverer's psychological reaction. In fact, researchers in cognitive psychology proposed a "surprise" heuristic for modeling this problem solving feature. We can understand this strategy as a way to find a solution. For example, according to Serratosa [23], in the field of chemical synthesis there is room for unplanned findings, and Woodward talks about "taking advantage of the surprises which may occur during the execution of a synthesis" [23] (p. 74). However, this way of presenting "blindness" blurs the differences between the satisfaction and search metaphors described earlier. If this difference is important, then we have to distinguish between a blind finding and a blind search. As a consequence, the above citations regarding chemical synthesis have to be considered as a sort of blind finding but not as a kind of blind search. It seems that the key difference here is not the structured context where the research is done, but the particular strategy used in some stage of the research. We can consider some states of the glutamine synthesis or the discovery of the ornithine cyle made by Krebs, as examples of a blind search.

Cognitive psychologists have studied those discoveries by Krebs and made computational reconstructions. In some of the reconstructions, initial strategies are depicted as "breadth-first" and then after the discovery as "depth-first" [13], using the idea of problem solving as a search in a tree of instances. A search of the first kind, "breadth-first" is usually useful when we do not have a lot of relevant information about the place (inside the "tree") where the solution is. Therefore, it is a kind of unrestricted search. On the other hand, a search is "restricted" to one of the "branches" of the tree when there is some positive indication that the solution is in some node below that branch.

As Frederick Holmes has pointed out, Krebs did not have relevant knowledge about what an intermediate metabolism mechanism should be. At that time, there were theories about the operation of the intermediate metabolism [9] that could have attracted a more trained scientist. Nevertheless, Krebs did not have enough knowledge to see the elegance of those theories [8]. "Having had less than a year of systematic training in chemistry, he did not possess the extensive knowledge of the 
properties and reactions of organic compounds necessary to reason deeply about the metabolic steps that would be most likely, on theoretical grounds, to take place. If his lack of expert knowledge in organic reactions made it difficult for him to choose the most promising theoretical possibilities in advance, it freed him at the same time from some of the biases built into the conceptual frameworks within which others operated. He became an investigative scanner, gazing back and forth across his experimental horizon for an unusual effect" [8] (p. 273).

Therefore, some stages of Krebs's search can be described as "blind" or "unrestricted" by previous knowledge. A search should be close to exhaustive - in a pragmatic sense- if we have no clues about the nature or place of where the solution is. Such a barely guided search could be very sensitive to any anomalous result: "His approach was one of trial-and-error search, of trying many things because he did not spend a lot of time working out in advance which ones had the best chance for success...; for it was, in a sense, forced on him by the absence in his repertoire, of heuristic strategies enough to guide him more directly toward solutions to his problems" [8] (p. 272).

If these considerations are correct, then we should be more careful when we evaluate blind or unrestricted searches. We should emphasize that such strategies are applied in a very restricted scientific context and if this strategy was successful in a scientific environment, within normal scientific parameters, we would consider it a heuristic rather than an algorithmic rule.

\section{Final words}

We began this paper by defining the "rational turn" in combinatorial chemistry as a departure from serendipity. In this sense, the first attempt in combinatorial synthesis is seen as a kind of "irrational" or "serendipitous" approach. This leads us to the question of whether or not the use of the word serendipity is correct or if, on the contrary, it is merely metaphoric and that when talking about serendipity, we can refer to different aspects of discovery. Taking into account the criticism made by the "rational turn" in combinatorial chemistry we identify the serendipity approach implicit in the "first attempts" as a type of "blind search". In this way, the "serendipitous mistake" in the first attempts in combinatorial synthesis was a blind or unrestricted search. We tried, then, to undermine the affirmation that a blind search is always irrational by showing how this kind of search could be a good strategy in a creative context and in particular scientific contexts.

Some may say that the notion of serendipity is or consists of "accidental" ways to find something, so the whole project of looking for a kind of "rationality" here is misleading. However, we tried to show that serendipity, as long as it is defined in an ostensive way, has different meanings, and when is described as a "serendipity mistake" in combinatorial chemistry is blind search and not accidental circumstances or even prepared minds. In this sense, we can assume as genuine the use of the notion of serendipity by those who criticized "the first approach". Particularly, this use of serendipity seems legitimate considering the particular type of search that would constitute "the serendipity part of some discoveries". We assert that the research 
carried out by the first intents in combinatorial chemistry were not - in any waysimply blind search. This label of "serendipity mistake", and its negative implications, only appears in the contrastive context was used by those who carried out more sophisticated searches through the so called "rational turn".

The question regarding what the notion of serendipity includes - and what it should include - is debatable. I have suggested that for the purpose of preserving descriptive capacity in relation to historical cases, they should include the aspects presented. However, those aspects alone are not sufficient to label something as serendipitous. Nonetheless the problem can now be considered in relation to what the expression "serendipity mistake" encompasses. What the scientists seem to have done is take a certainly genuine aspect of the notion of serendipity- blind search- and isolating it to make reference to a methodological strategy. In this sense, the discussion of the expression "serendipity mistake" seems justified in methodological terms. However, this problem should be considered with a certain independence from that which refers to the possibility of recovering a certain kind of blind search within scientific practices, and in this way we have enough to evaluate the precise reach of the critiques of those who are working in combinatorial chemistry ${ }^{4}$.

\section{Acknowledgment}

I would like to thank two anonymous referees and especially to the editor for their helpful and thoughtful comments on previous versions of this paper.

I would also like to thank FONCYT for the research grant that supported the work of this paper, as well as Secyt (Universidad Nacional de Cordoba).

\section{References:}

\footnotetext{
4 It could be argued also that if we understood the relationship between 'irrational' and 'rational' assumed by the "rational turn" in combinatorial chemistry as a continuum, then we could only speak about a "less rational" strategy and not about an "irrational" strategy. There is also a more radical version of this criticism. The discussion in combinatorial chemistry is not about rationality but about "effective" or "ineffective", so the rational turn has to be interpreted in a pragmatic way, not in an "epistemic" way. The common reference to the amount of money invested in those techniques and devices as a relevant parameter supports this last view. Nevertheless, according to the examples, the main point is not about rationality itself: it is about which strategy we are allowed to use and whether or not searching in a 'blind' way is a wise choice. It could also be argued that a blind search for drug discovery is a good strategy only if the drugs are distributed in a homogenous way in the space to be searched. This last assumption seems to be false, so a blind search would only be a waste of time. However, we do not suppose that a "blind strategy" could be considered a regular strategy. If there is a relationship between serendipity and creativity, then we have to assume that a blind search is not a common path. Consequentially, we cannot recommend a blind search for normal research, but we can use it as an explanation for some particular and very rare events in science.
} 
1. Bayley, M. Gillet, V. and Willett, P. Computational Analysis of Molecular Diversity for Drug Discovery, RECOMB, ACM (1999).

2. Bajorath, J. (edit) Chemoinformatics: Concepts, Methods, and Tools for Drug Discovery, Vol. 275. Humana Press, 2004

3. Booth; Marijke Dreef-Tromp; Hermkens, de Man; Ottenheijm Survey of SolidPhase Organic Reactions in Jung, G (1999).

4. Bleicher, H. Konrad, et al. Hit and Lead Generation: Beyond High-Throughput Screening. Nature Reviews, Drug Discovery. 2003 May:(2), 369-378.

5. Campbell, D. "Evolutionary Epistemology." In The philosophy of Karl R. Popper edited by P. A. Schilpp, 412-463. LaSalle, IL: Open Court. (1974).

6. García, P. "Interaction between History of Science and Automatic Computational Discovery: A Two Way Road", in "Interdisciplinary aspects of Human-Machine Co-existence and Co-operation" (Proceedings) Marik, V.; Jacovkis, P; Stepanokova, O.; Klema, J. (edits), Czech Technical University, Prague (2005).

7. Hoffmann, R. The Same and Not the Same, Columbia University Press, New York, (1995).

8. Holmes, F., Hans Krebs: The formation of a Scientific Life 1900-1933, Vol. 1. Oxford University Press, Oxford. (1991).

9. Horrobin, D. Innovation in pharmaceutical industry, J R Soc Med; 93: 341-345. (2000).

10. Huser, J. High-throughput screening in drug discovery Weinheim : Wiley-VCH, c2006. (Series: Methods and principles in medicinal chemistry ; v. 35)

11. Jung, G. "Combinatorial Chemistry", Wiley-VCH, Verlag (1999).

12. Kantorovich, A. Scientific discovery: logic and tinkering. Albany: State University of New York Press. (1993).

13. Kubinyi $\mathrm{H}$. Chance favors the prepared mind-from serendipity to rational drug design. J. Recept Signal Transduct Res. 1999; 19: 15-39.

14. Kulkarni, D.; Simon, H.A. "The processes of scientific discovery: The strategy of experimentation" Cognitive Science, 12, 139-175. (1988).

15. Lamb, C. and Perun, Thomas J., (editors), Nucleic Acid Targeted Drug Design, M. Dekker, New York, (1992).

16. Leach, A.; Hann, M. The in silico world of virtual libraries, DDT Vol. 5, No 8, August (2000).

17. Leach, A; Gillet, V. "An Introduction to Chemoinformatics", Kluwer Academic Publishers (2003).

18. Merrifield, Automated Synthesis of Peptides: solid-phase peptide synthesis, a simple and rapid synthetic method, has now been automated, Science, vol. 150 (Oct. 8) pp. 178-185. (1965).

19. Merton, R., and Barber, E. The Travels and Adventures of Serendipity: A Study in Sociological Semantics and the Sociology of Science. Princeton, NJ: Princeton University Press, (2004).

20. Roberts, R. Serendipity: Accidental Discoveries in Science, New York: Wiley, (1989).

21. Salemme FR, Spurlino J, Bone R. Serendipity meets precision: the integration of structure-based drug design and combinatorial chemistry for efficient drug discovery. Structure. (1997) Mar 15;5(3):319-24.

22. Sanders, J. "Polymer libraries obtained with combinatorial techniques expedite and improve design and testing of new materials" Georgia Institute of Technology, (2004).

23. Serratosa, F. and Xicart, J. Organic Chemistry in Action (Studies in Organic Chemistry), Elsevier Science, (1996). 
24. Van Andel, P. "Anatomy of the unsought finding: serendipity: origin, history, domains, traditions, appearances, patterns and programmability." British Journal for the Philosophy of Science, 1994, 45(2), 631-648. 